\title{
GAMMA DECAY OF ISOSCALAR AND ISOVẼCTOR GIANT RESONANCES \\ FOLLOWING HEAVY-ION INELASTIC SCATTERING
}

J. R. BEENE, R. L. VARNER, and F. E. BERTRAND

Dak Ridge National Laboratory

Oak Ridge, Tennessee 37831, U.S.A.

\author{
Invited Paper Presented at the \\ First Topical Meeting on \\ Giant Resonance Excitation in Heavy-Ion Collisions \\ Legnaro, Ita $1 y$ \\ September 21-25, 1987
}

\section{DISCLAIMER}

This report was prepared as an account of work sponsored by an agency of the United States Government. Neither the United States Government nor any agency thereof, nor any of their employees, makes any warranty, express or implied, or assumes any legal liability or responsibility for the accuracy, completeness, or usefulness of any information, apparatus, product, or prociss disclosed, or represents that its use would not infringe privately owned rights. Reference herein to any specific commercial product, process, or service by trade name, trademark, manufacturer, or otherwise does not necessarily constitute or imply its endorsement, recommendation, or favoring by the United States Government or any agency thereof. The views and opinions of authors expressed herein do not necessarily state or reflect those of the United States Government or any agency thereof. 
J. R. BEENE, R. L. VARNER, and F. E. BERTRAND

Oak Ridge National Laboratory, " Oak Ridge, Tenriessee 37831, U.S.A.

Results from studies of gamma decay of giant resonances in $200 \mathrm{~Pb}$ and $90 \mathrm{Zr}$ following inelastic excitation by 22 and $84 \mathrm{MeV} /$ nucleon ${ }^{27} 0$ ions are presented. Data on ground state decay of isoscalar giant quadrupole and isovector giant dipole resonances are presented and compared with calculations. Decays from resonances to low lying excited states are also discussed. Preliminary results from an attempt to isolate the isovector quadrupole resonance in $20 \mathrm{sb}$ using its gamma decay are presented.

Coincidence experiments designed to investigate decay properties now make up a significant part of the experimental study of giant resonances. Since most giant resonances (GR) lie above the particle emission threshold the doninant decay mode is usually nucleon or alpha emission (neutron emission in heavy nuciei) with gamma decay having a small $\left(\leqslant 10^{-3}\right)$ probability. Nucleon emission can in principle carry important information about the microscopic structure of resonances, damping mechanisms and damping rates; a number of such studies will be reported at this meeting. Photon decay studies face difficulties resulting from the small probability of such events, yet they too can provide significant information, often illuminating asperts of the GR different from those probed by nucleon decays. In his presentation ${ }^{1}$, Fred Bertrand has illustrated the large GR cross sections and resonance to continuum ratios which can be achieved with inelastic scattering of selected heavy ions at energies above $\sim 20$ MeV/nucleon and emphasized the rapid increase in both these quantities with increasing bombarding energy. Study of low probability events like photon decay can benefit in obvious ways from these characteristics.

Photon decay data can be extremely sensitive to GR multipolarity - thus compensating for a principal weakness in heavy ion scattering. For example, gamma decay back to the ground state following heavy ion scattering is almost certain to be dominated by the isovector giant dipole resonance (IVGDR). Under very favorable conditions ground state decay of giant quadrupole resonance (GQR) can also be observed - but in the 10 to $25 \mathrm{MeV}$ excitation energy region

\footnotetext{
*Operated by Martin Marietta Energy Systems, Inc. under contract DE-AC05-840R21400 with the U.S. Department of Energy.
}

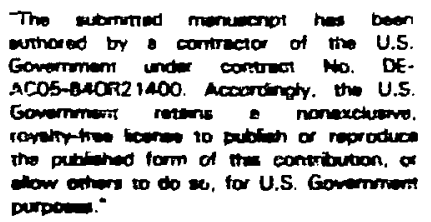


nigher multipolarities are extremely unlikely to coiltribute to the g.s. decay. This is illustrated in figure 1 , which shows the ground state gama width ( $\Gamma_{\text {Yo }}$ ) expected for a sharp state exhausting $100 \%$ of the relevant 1 soscalar (IS) or isovector (IV) energy weighted sum rule (EWSR) as a function of multipolarity and energy, relative to that for the GDR. Ground state garma decay can also yield data on electromagnetic strength of resonances, and provides simple, well-defined conditions under which we can investigate the multistep theory of nuclear reactions in terms of which GR decay is conventionally discussed.

Photon decays from the GR to low lying excited states are also poicential sources of significant information. These data, like ground state decays, provide significant multipole selectivity - but are not 1 imited to $L=1$ and 2 resonances. Decays to excited states, like ground state decay, are dominated by El transitions. Thus, for example, transitions from the GR region to a low lying $5^{-}$state in an even-even nucleus is clear evidence for high spin $4^{+}$or $\left.6^{+}\right)$strength. Decays to low lying collective states can provide important information about the coupling of (isovector) GR modes to these low frequency collective modes. Recent calculations ${ }^{2,3}$ have shown that $E 1$ transitions between isoscalar GR and low lying collective states (also isoscalar) can be strongly suppressed. Thus study of such transitions can provide important data on the isospin character of resonances. In this talk we will illustrate some of these asfects of gamma decay studies with experimental data, and try to convince you that such experiments have great potential at intermediate energy heavy ion facilities.

Figure 2 shows a spectrum of inelastically scattered ${ }^{17} 0$ ions obtained with the ${ }^{208} \mathrm{~Pb}\left({ }^{17} 0,{ }^{17} \mathrm{O}^{\prime}\right)$ reaction at $84 \mathrm{MeV} /$ nucleon in a recent experiment carried out at GANIL in collaboration with scientists from GANIL, Saclay, and Strasbourg. The ${ }^{17} 0$ ions were detected and analyzed in the energy loss magnetic spectrometer SPEG, set up to cover the angular range $1.5^{\circ}$ to $5^{\circ}$. A detailed analysis of these singles data has been discussed at this conference ${ }^{1}$ and elsewhere ${ }^{4}$; here I merely note that the cross section in the GR region ( 9 to $20 \mathrm{MeV}$ of excitation) is on the order of 5 barns/sr with over half that res"iting from Coulomb excitation of the IVGOR! Figure 3 is identical io figure 2 except that a coincidence with a single garma ray to the ground state was required (i.e., with $E_{y}=E^{*}$, where $E^{*}$ is the excitation energy). Both figures 2 and 3 correspond to a broad range of ${ }^{17} 0$ angles from $2^{\circ}$ to $3.5^{\circ}$. Gamma rays were detected in four groups of seven Baf $z$ crystals (individually hexagons with face to face diameter $8.7 \mathrm{~cm}$ and length $14 \mathrm{~cm}$ ) positioned at angles $(\theta, \phi)$ of $\left(95^{\circ}, 0^{\circ}\right),\left(70^{\circ}, 180^{\circ}\right),\left(134^{\circ}, 180^{\circ}\right),\left(90^{\circ}, 270^{\circ}\right)$. The angles are defined in the conventional way with respect to a coordinate system with the 
$z$ axis along the beam and the $y$ axis perpendicular to the reaction plane. The gamma coincidence spectrum shows prominent structures corresponding to well known low lying states which have strong ground state gamma branches, notably the $2.6 \mathrm{MeV} 3^{-}, 4.08 \mathrm{MeV} 2^{+}$states, and the $5.512 \mathrm{MeV} 1^{-}$states. Gama anguiar distributions demonstrate that most of the yield between the $5.5 \mathrm{MeV}$ state and the neutron separation energy (7.4 MeV) corresponds to $1^{-}$states (probably the 7.06 and $7.08 \mathrm{MeV} 1^{-}$states), but a $2^{+}$state, at $\sim 6.2 \mathrm{MeV}$ is also present. Above the neutron separation energy the cuincident yield falls rapidly, but rises again to a strong broad peak in the GR region. The coments made earlier, together with the large cross sections for the GDR (see Ref. 1), would lead us to expect the 9 to $20 \mathrm{MeV}$ region of the gamma coincidence spectrum to be dominated by the IVGDR. This is illustrated qualitatively in figure 4 . The heavy solid line is the GR peak from the data in figure 2. The short dashed curve is the distribution of IVGDR cross section calculated assuming pure coulomb excitation, and utilizing the strength distribution for the GDR from photonuclear experiments. The histogram is the gama coinciderice spectrum, arbitrarily normalized. Clearly, the ground state gama coincidence effectively selects the IVGDR from the complex multipole distribution illustrated in figure 16 of Ref. 1. This fact is illustrated more quantitatively in figures 5 and 6 which show ${ }^{17} 0$ - $y_{0}$ angular correlations. In figure 5 the correlation is displayed as a function of 170 angle, for ground state gammas at $\theta=90^{\circ}$, $\phi=270^{\circ}$, while in figure 6 the data are displayed as a function of $\gamma$ angle for the ${ }^{17} 0$ ions detected in the range $\theta\left({ }^{17} 0\right)=2^{\circ}$ to $3.5^{\circ}$. In this case the angle $\theta_{y}$ is referred to the direction of the $20 \mathrm{pb}$ recoil rather than the beam axis. The lines on figures 5 and 6 are calculations carried out with the code ECIS ${ }^{5}$, assuming pure Coulomb excitation of the IVGDR with total GDR strength and its distribution taken from Ref. 9. The calculation of the absolute theoretical cross sections in figure 5 involve an average ground state gamma branching ratio, which is, of course, not provided by the ECIS calculation. For now this branching ratio can be regarded as determined by fitting the overall normalization in figure 5 to be $0.017 \pm 0.002$, however, we will see later that this quantity is in excellent agreement with a calculation containing no free parameters.

Al1 GDR caiculations shown assume pure Coulomb excitation. Nuclear excitation of the GDR can play a role primarily through terms arising from the fact that both projectile and target have a neutron excess. Calculations employing a nuclear transition density calculated following the methods of Satchler ${ }^{6}$, demonstrate that the nuclear contribution to the cross section of the IVGDR is about $10^{3}$ times smaller than the coulomb, and has no significant effect on any of the calculations presented here. 


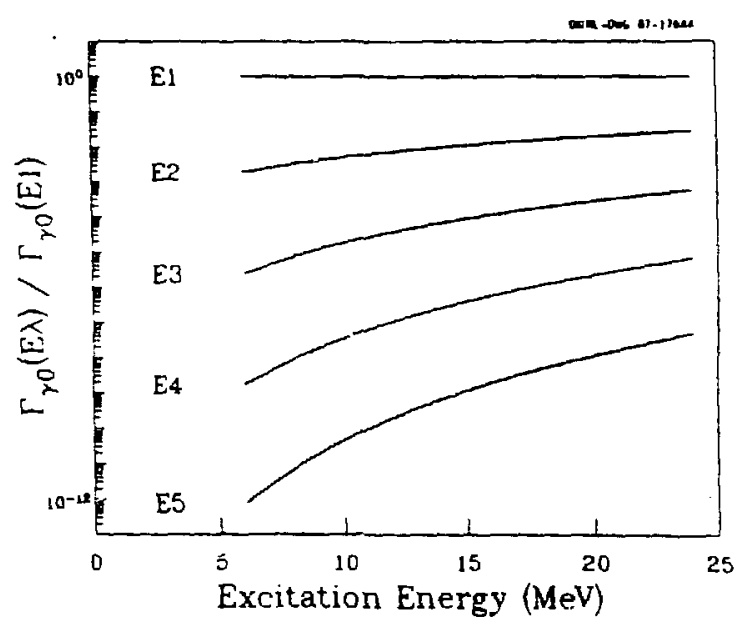

FIGURE 1

Ground-state gamma widths of hypothetical sharp states fully exhausting the appropriate isovector or isoscalar energy weighted sum rule as a function $:$ the excitation energy of the state, relative to the El width.

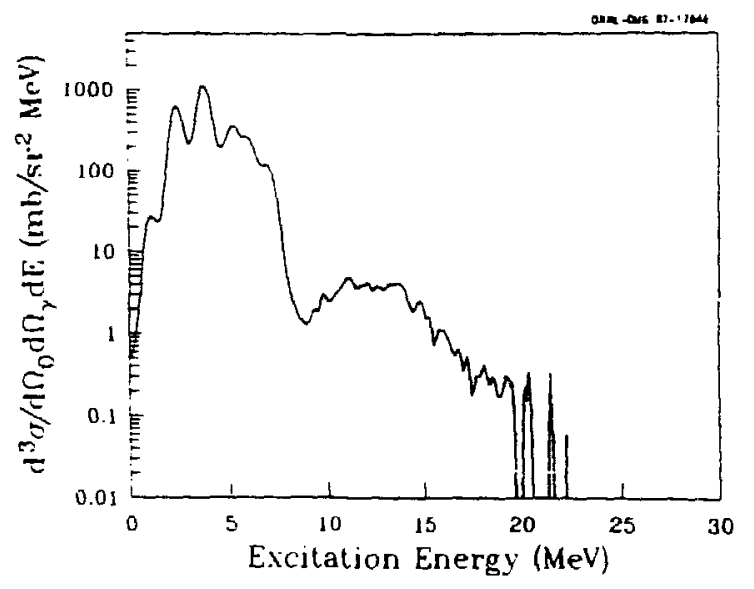

FIGURE 3

Ground stati gamma-ray coincidence spectrum. The same spectrum as figure 2 , but in coincidence with gamma rays to the ground state. (The ${ }^{17} 0$ angles are $\theta=2.0^{\circ}-3.5^{\circ}$ in this case, the gamma detector angles are given in the text.)

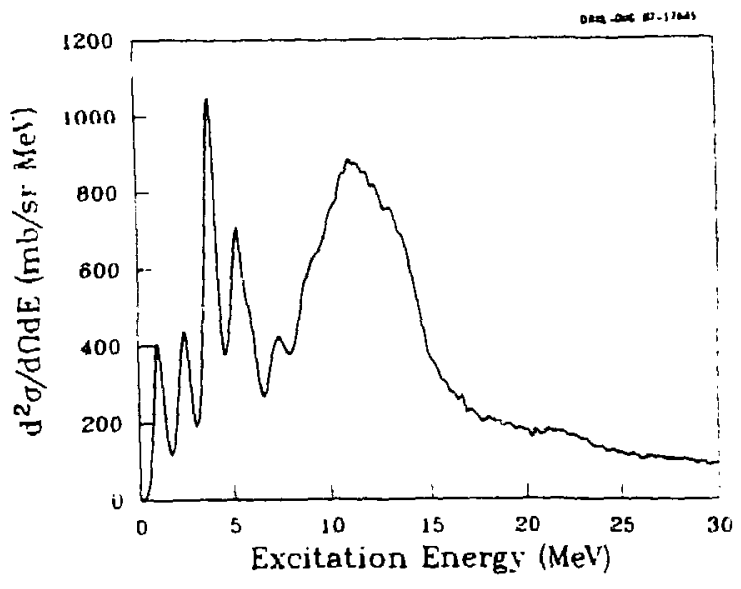

FIGURE 2

Spectrum of ${ }^{17} \mathrm{O}$ ions from the $208 \mathrm{~Pb}\left({ }^{17} 0,{ }^{17} 0^{\prime}\right)$ reaction at 84 MeV/nucleon. The ${ }^{17} 0$ was detected over the range $\theta=2.0^{\circ}-3.0^{\circ}$.

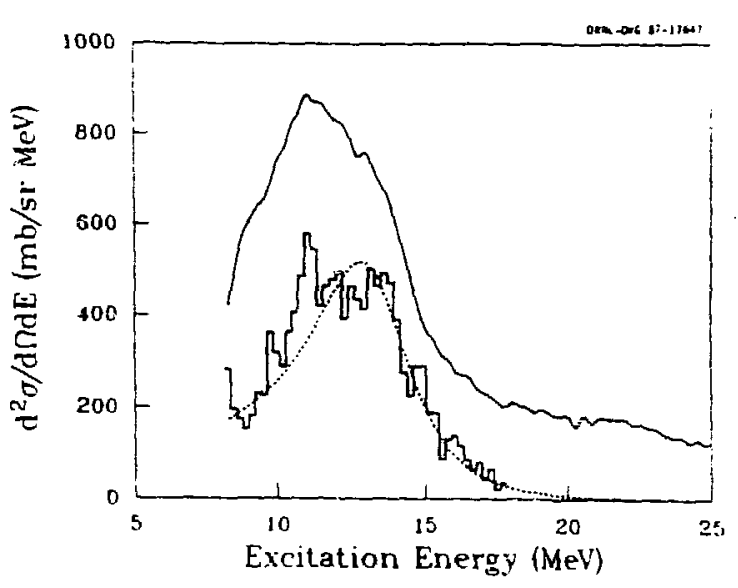

FIGURE 4

Multipole selectivity by garma coincidence. The heavy solid line is the giant resonance region of the data in figure 2 . The dashed line is the theoretical contribution of the giant dipole resonance to the experimental spectrum. The histogram is the ${ }^{17} \mathrm{O}-\mathrm{Y}_{0}$ coincidence data of figure 3 . 
The absolute yield of ground state gamma rays can be calculated from the properties of the GDR by applying the ideas of the multistep theory of nuclear reactions ${ }^{7,8}$. The collective $1 P-1$ h GR state is considered as a doorway state which couples strongly to the inelastic scattering process. This state damps into the more complex $2 p-2 h, 3 p-3 h$, etc. states eventually reaching the fully damped compound states. The cross section for emission of ground-state gamma rays following inelastic scattering can be expressed as

$$
\sigma_{x, X^{\prime} Y_{0}}(E)=\sigma_{x, X^{\prime}}(E)\left(\sum_{i=1}^{r} \frac{\Gamma_{Y_{0}, j}}{\Gamma_{i}}\left[\prod_{j=1}^{i-1} \frac{\Gamma_{j}^{+}}{\Gamma_{j}}\right]\right)
$$

$\sigma_{x, x^{\prime}}(E)$ is the distribution of excitation cross section obtained from a DWBA calculation, the sum in parentheses runs over the hierarchy of levels of complexity from the doorway stage $(i=1)$ to the compound $\left(r^{\text {th }}\right)$ stage. The quantity $\Gamma_{j}^{\downarrow}$ represents the damping width of the $i$ th stage while $\Gamma_{i}$ is the total width. Application of this general expression requires a great deal of knowledge concerning the various widths associated with each stage. For simplicity and without much justification, we will use a two stage approximation, considering only the GR doorway and the compound states.

$$
\sigma_{x, x^{\prime} y_{0}}(E)=\sigma_{x, x^{\prime} y_{0}}(E)\left(\frac{\Gamma y_{0}}{\Gamma}+\left[\frac{\Gamma^{\downarrow}}{\Gamma}\right] B_{C N}(E)\right)
$$

where the index 1 for the doorway state has been dropped. $\Gamma_{\text {yo }}$ can be calculated directly from the GR strength, $\Gamma$ is identified with the experimental width of the resonance, and $B_{C N}(E)$ is the compound nucleus branching ratio. The quantity in square brackets assures that only that fraction of systems which survive the damping process ( $\Gamma^{\star}$ is the damping width) is included in the compound term. Theoretical and experimental results for $208 \mathrm{pb}$ indicate the $\Gamma^{\downarrow} \geq 0.9 \Gamma$, so that this factor can be considered as introducing an uncertainty of up to $10 \%$ in the compound contribution (we will set it to unity). The compound branching ratio can be calculated from mean $V_{0}$ and total widths obtained from Hauser-Feshbach calculations ${ }^{10,11}$, but including a correction for effects due to the distribution of partial widths which have been discussed extensively in the literature ${ }^{12}$ : i.e.,

$$
B_{C N}=C \frac{\left\langle\Gamma_{Y_{0}, C N}\right\rangle}{\left\langle\Gamma_{C H}\right\rangle}
$$


where $C$ is calculable in a straight forward way ${ }^{12}$ if we assume the partial widths have a Porter-Thomas distribution. The results of a calculation using Eq. (2), and the properties of the IVGDR $\left(\Gamma=4.0 \mathrm{MeV}\right.$ and $E_{0}=13.5 \mathrm{MeV}$ and strength $=100 \%$ of classical EWSR between 8 and $25 \mathrm{MeV}$ ) from Ref. 9 are compared with $\gamma$-angle-averaged experimental data in figure 7 . The contribution of the first and second terms of Eq. (2) are shown separately as dashed and dashdotted curves respectively, and the sum as a solid line. The branching ratio, integrated over excitation energy from 9.5 to $25 \mathrm{MeV}$, is found to be 0.016 , in excellent agreement with the value $0.017 \pm 0.02$ found earlier in connection with the data in figure 5. Furthermore the distribution of Yo yield as a function of excitation energy is reproduced very well.

Inelastic scattering of heavy ions at lower energies generaliy excites isovector resonance states very weakly. We have studied the ground state gamma decay of the giant resonance region following the ${ }^{208} \mathrm{~Pb}\left({ }^{17} 0^{17}{ }^{1} \mathrm{O}^{\prime}\right)$ reaction at $22 \mathrm{MeV} /$ nucleon using the Spin Spectrometer at Oak Ridge. Experimental details can be found elsewhere 13,14 . At this energy the inelastic singles spectrum $\left(\theta(170)=13^{\circ}\right)$ is dominated by the isoscalar giant quadrupole resonance (ISGQR), with a cross section of about $40 \mathrm{mb} / \mathrm{sr}$ while the IVGDR is calculated to have a cross section $<3 \mathrm{mb} / \mathrm{sr}$. In figure 8 the total ground state gamma coincidence spectrum is shown. Comparison with figure 3 shows the much smaller yield of high energy garma radiation in this case. Figure 9 shows the GR region of figure 8 , compared with a calculation identical to that discussed above in connection with figure 7 . In figure 9a the total experimental spectrum is shown compared to a calculation including only the IVGDR, which, in spite of its weak excitation is clearly dominant. In figure 90 data obtained by subtracting the calculated GDR contribution from the experimental spectrum is compared with a calculation for the ISGQR gamma decay. The GQR resonance parameters are taken from high resolution $\left(p, p^{\prime}\right)$ data ${ }^{15}$. In both figure $9 a$ and $3 b$ the doorway and compound terms are shown separately as dash-dot and shortdashed curves respectively, while the heavy solid lines are the sum. The liant solid lines in figure 9 a represent the tail of the yield from around the neutron separation energy, while in figure 90 the light solid line represents the contribution of narrow $2^{+}$states found at 8 and $9.3 \mathrm{MeV}$ in $\left(p, p^{\prime}\right)^{15}$. The gamma ray angular correlation data shown in figure $9 c$ confirms the presence of E2 gamma radiation in the 9 to $11 \mathrm{MeV}$ region and agrees very well with the relative E2 and E1 contribution predicted by the calculations. From the data in figure 9b we obtain a total E2 ground state cross section of $17 \pm 4 \mu \mathrm{b} / \mathrm{sr}$, corresponding to a total branching ratio for the GQR of $(4.1 \pm 1.0) \times 10^{-a}$ or an electromagnetic reduced matrix element of $B(E 2 \uparrow)=(6.2 \pm 1.2) \times 10^{3}$ 


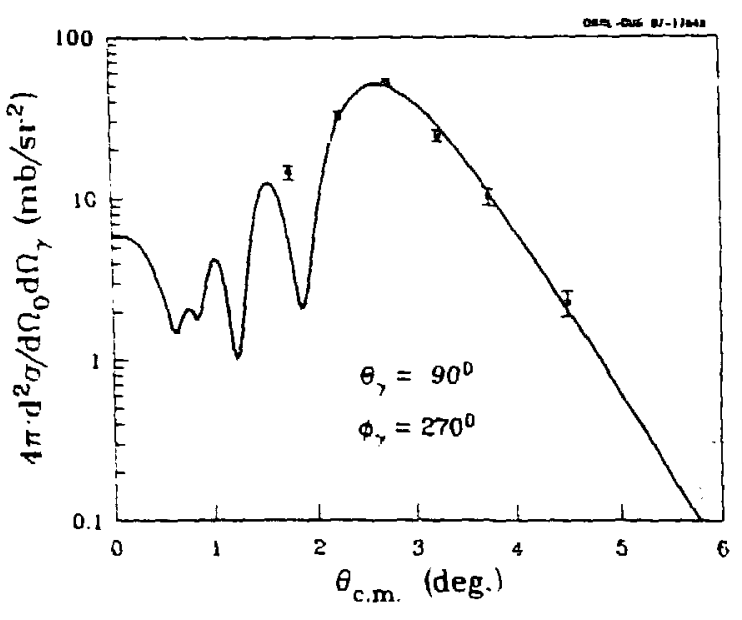

FIGURE 5

$170-Y_{0}$ angular correlation for the $208 \mathrm{~Pb}\left({ }^{17} 0,1^{17} 0^{\prime}\right)$ reaction at 84 $\mathrm{MeV} / \mathrm{nucleon}$, for fixed $y$ angle $\theta_{y}=90^{\circ} \phi_{y}=270^{\circ}$.

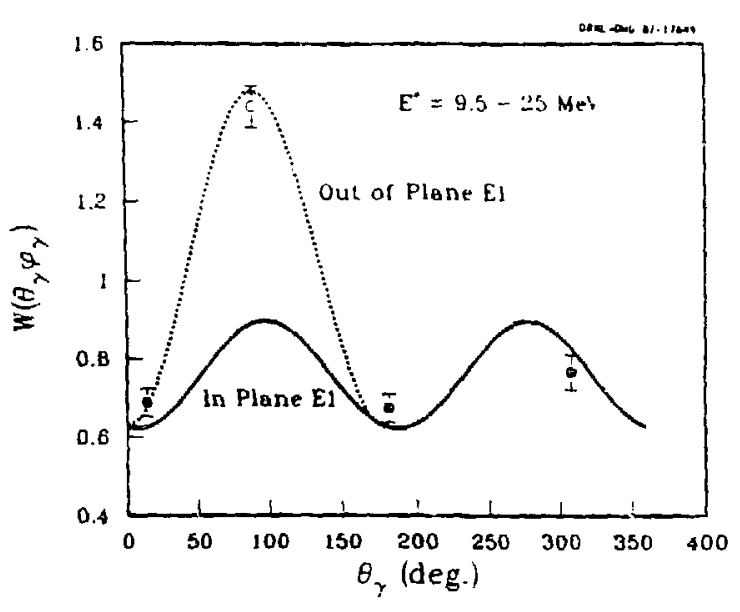

FIGURE 6

The same correlation as figure 5 , but for fixed ${ }^{17} 0$ angle $\left(\theta=2^{\circ}-3^{\circ}\right)$ and varying $\gamma$ angle. The lices are from theoretical calculations assuming pure Coulomb excitation of the GDR. Filied data points and the solid curve lie in the reaction plane $\left(\phi=0^{\circ}\right.$ and $180^{\circ}$ ). (For convenience the $\phi=180^{\circ}$ half plane is labeled by $\theta+180^{\circ}$.) The open point and dashed line refer to the $\phi=270^{\circ}$ half plane.

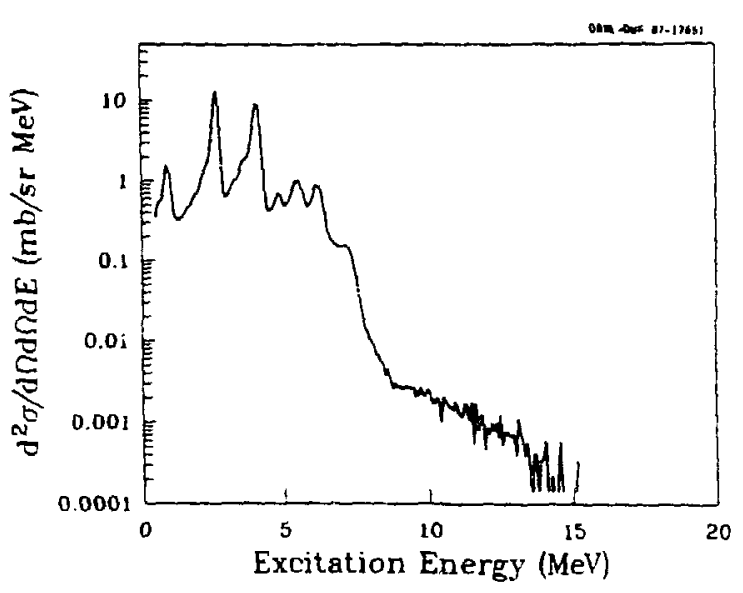

FIGURE 8

The angle-integrated ground-state gamma ray cross section in coincidence with inelasticaily scattered 170 ions at $\theta=13^{\circ}$, for 2aspb(170,170') at $22 \mathrm{MeV} / \mathrm{nucleon}$.

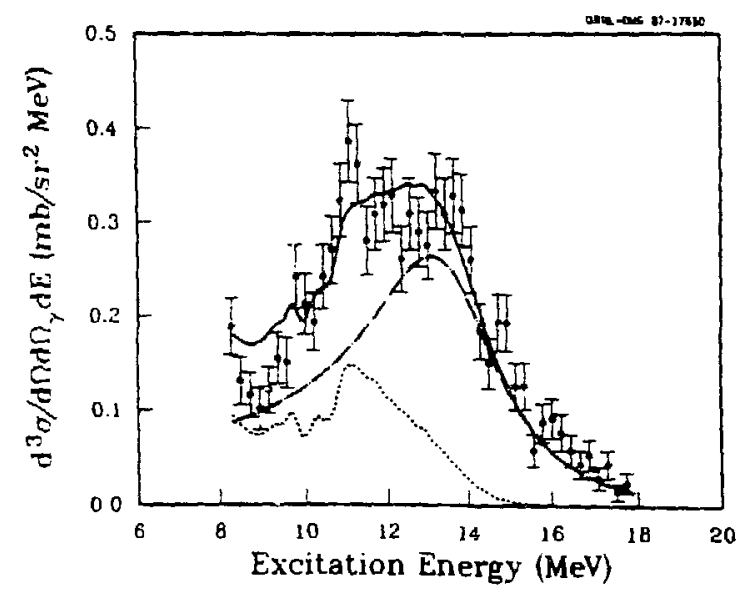

FIGURE 7

The ground state gama coincidence yield for $84 \mathrm{MeV} /$ nucleon 170 scattering on $20 \mathrm{~Pb}$, compared with calculations as discussed in the text. 
$e^{2} \mathrm{fm}^{4}$ for the $10.6 \mathrm{MeV}$ ISGQR. This corresponds to $87 \pm 20 \%$ of the strength expected for a state exhausting the full E2 EWSR, assuming the ratio of neutron to proton matrix elements in the $I S G Q R$ is $M n / M p=N / Z=1.5$. An experimental value for the ratio $M_{n} / M_{p}$ can be deduced from our $B(E 2 t)$ if the cross section for alpha-particle inelastic scattering ${ }^{16}$ is used as a measure of $\left(M_{n}+M_{p}\right)^{2}$. We obtain $M_{n} / M_{p}=1.35 \pm 0.33$ in good agreement with the value $N / Z$ expected for an approximately isoscalar GR having equal neutron and proton deformation. Electron scattering results ${ }^{17}$ can be combined with $\left(\alpha, \alpha^{\prime}\right)$ results in a similar way to obtain $M_{n} / M_{p}-1.75 \pm 0.4$, again in good agreement with $N / Z$. These results disagree with the small value of $B(E 2 t)=(1.01 \pm 0.6) \times 10^{3} \mathrm{e}^{2} \mathrm{fm}^{6}$ and $M_{n} / M_{p}=3.8$ deduced from recent $\pi^{+}$and $\pi^{-}$scattering data. These quantities would require that the $10.6 \mathrm{MeV} G Q R$ in $208 \mathrm{~Pb}$ has a strongly mixed isospin character.

Data for ground state gamma decay produced in the ${ }^{\circ} \mathrm{Zr}\left({ }^{17} 0,{ }^{17} 0^{\prime} Y_{0}\right)$ reaction at $22 \mathrm{MeV} /$ nucleon are shown in figure 10. Comparison with figure 8 shows that the absolute $Y_{0}$ yield is an order of magnitude smaller in this case compared to $208 \mathrm{~Pb}$. These results are, again, accounted for very well by calculations based on Eq. (2) like those carried out for ${ }^{20} \mathrm{~Pb}$. This is illustrated by figure 11. It has been realized for some time that gamma decay of GR to low lying collective states should carry important information about the coupling of low frequency surface vibrations to the GR. Such experiments on the IVGDR are an important part of the research program at tagged photon facilities. We have studied the branching to low lyifig excited states in $208 \mathrm{~Pb}$ as a function of excitation energy between $\sim 8.5$ and $16 \mathrm{MeV}$ in our $22 \mathrm{MeV} /$ nucleon 170 scattering experiments at Oak Ridge. The results have been discussed extensively elsewhere 13,14 . Table I summarizes the data obtained for a 2 MeV wide bin centered on the ISGQR. A particulariy interesting feature is the absence of any significant decay directly to the $3^{-}$state at $2.6 \mathrm{MeV}$. Purely statistical arguments suggest that this branch from the region of the GQR should be approximately equal to the ground state branch. Clearly it is strongly suppressed. The only decays from the GQR region strong enough to be clearly identified are a branch to the $4.9 \mathrm{MeV} 3^{-}$and a decay to the $5.512 \mathrm{MeV}$ $1^{-}$state. The latter is grouped in Table 1 with decays to a number of other states tentatively identified with known $1^{-}$levels between 5 and $7 \mathrm{MeV}$. The $5.5 \mathrm{MeV}$ state accounts for about $60 \%$ of this yield. Decay to the $5^{-}$state at 3.2 MeV was seen in the 8.5-9.5 MeV excitation energy bin and weaker evidence for decay to the $5^{-}$state at $3.9 \mathrm{MeV}$ in the 9.5-10.5 MeV bin was found, confirming the presence of $4^{+}$or $6^{+}$strength in these regions. In the decay of the 12.5-15.5 MeV excitation region, the only lines identified correspond to 


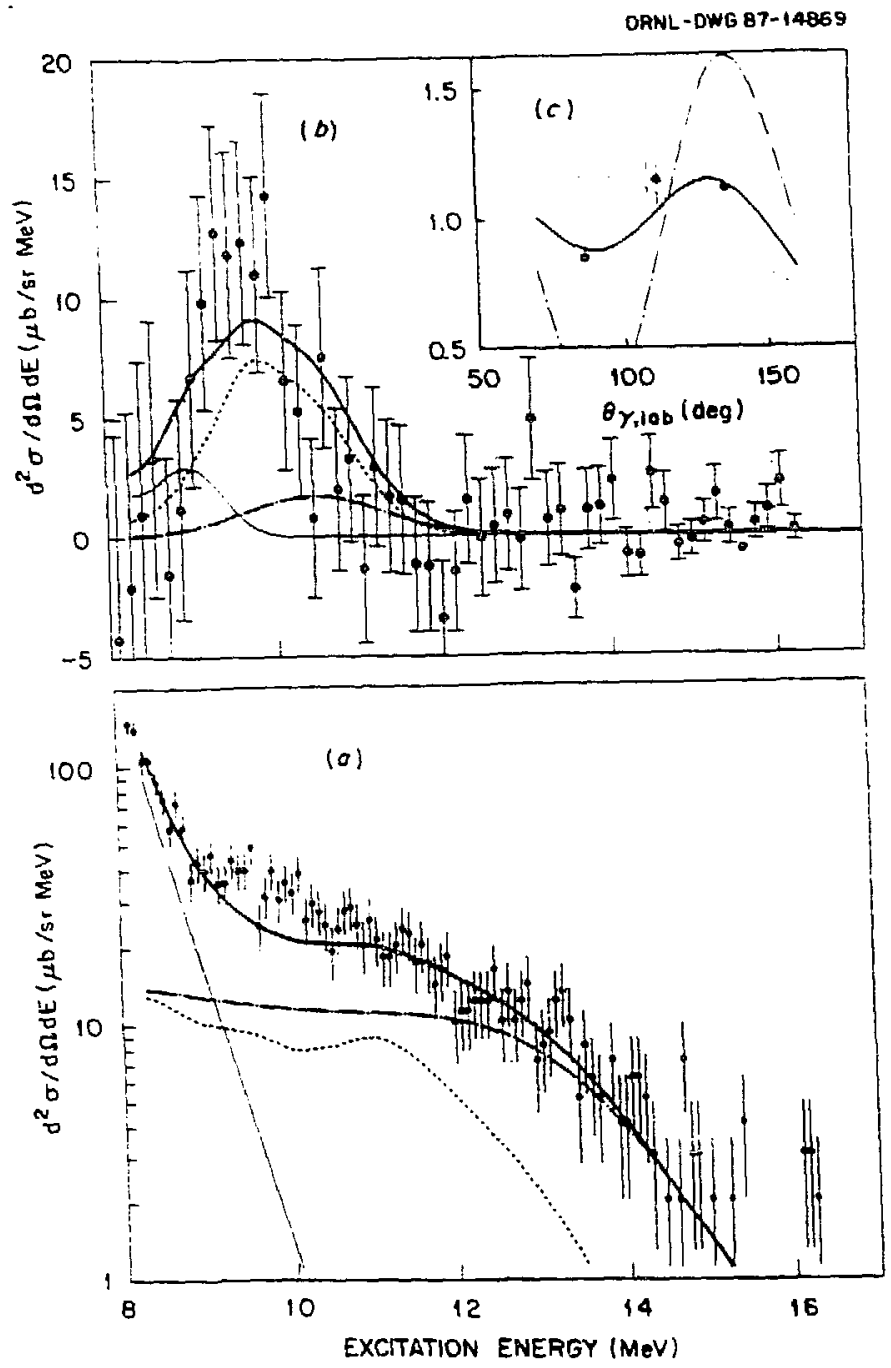

FIGURE 9

Ground state gamma ray coincidence data compared with calculations using Eq. (2). This is a subset of the data in figure 8 . The data and calculations shown in figure $9 a$ and $9 b$ are discussed in the text. Figure $9 c$ shows experimental $Y_{0}$ angular correlations for energy bins from 9-11 MeV (solid points) and 12-15 MeV (open points). The dashed curve is for pure El decay of the IVGDR. The dash-dot curve is for pure E2 decay of the ISGQR while the solid curve represents the $E 1+E 2$ mixture predicted at 9-11 MeV by the calculations shown in a and $b$.

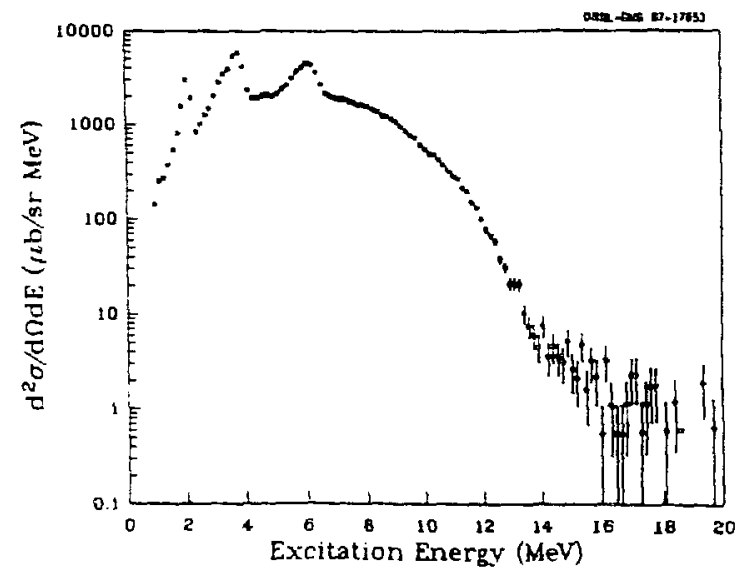

FIGURE 10

The same as figure 8 , but for the reaction ${ }^{\circ} \operatorname{Zr}\left({ }^{17} 0,{ }^{17} 0^{\prime}\right)$ at $\theta\left({ }^{17} O\right)=8.9^{\circ}$ and bombarding energy $22 \mathrm{MeV} /$ nucleon. (Note different cross section units than on Fig. 8.)

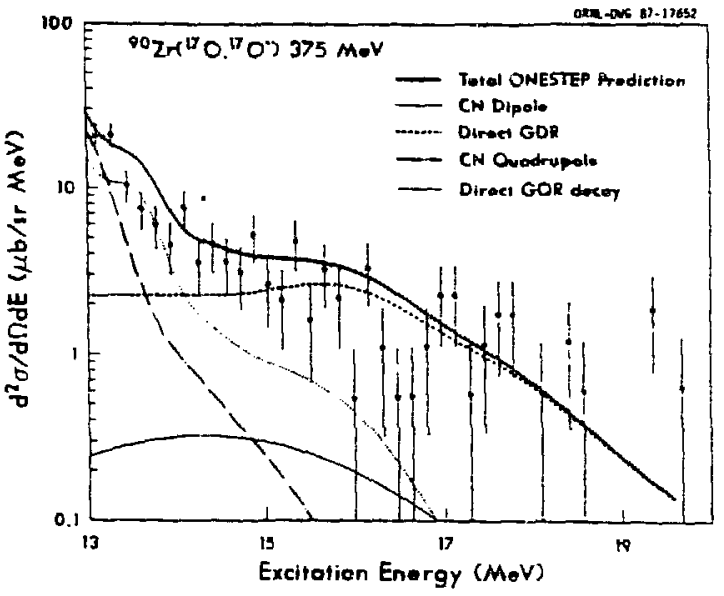

FIGURE 11

The giant resonance region of the data in figure 10, compared with theory as discussed in the text. 
TABLE I. Relative photon branching to low-lying states in $208 \mathrm{~Pb}$ from the excitation energy region of 9.5-11.5 MeV.

\begin{tabular}{|c|c|c|c|c|}
\hline \multicolumn{2}{|c|}{ Final state } & \multicolumn{3}{|c|}{ Decay branch relative to $\mathrm{g.s}$. } \\
\hline \multirow[t]{2}{*}{$E_{x}(\mathrm{MeV})$} & \multirow[t]{2}{*}{$3 \pi$} & \multirow[t]{2}{*}{ Experiment } & \multicolumn{2}{|c|}{ Calculations } \\
\hline & & & $R=? \quad=7$ & Ref. $\because$ \\
\hline 0.0 & $0^{+}$ & 1.0 & 1.0 & 1.0 \\
\hline 2.61 & $3^{-}$ & $0.04 \pm 0.04$ & 0.027 & 0.035 \\
\hline 4.085 & $2^{+}$ & $\begin{array}{l}0.02+0.05 \\
-0.02\end{array}$ & & $9 \times 10^{-3}$ \\
\hline 4.97 & $3-$ & $1.80 \pm 0.50$ & & $2.3^{\mathrm{a}}$ \\
\hline $5-7$ & $1^{-}$ & $1.50 \pm 0.50$ & & 0.34 \\
\hline
\end{tabular}

$1^{-}$states at 5.51,7.06, and/or $7.08 \mathrm{MeV}$. This would be consistent with the photon decay mode expecteu from the giant monopole resonance which dominates the excitation cross section in this region.

The strong suppression relative to statistical estimates of decays from the GQR region to the $2.6 \mathrm{MeV} 3^{-}$state is very interesting. Two recent calculations 2,3 (Table I) predict this suppression. In both calculations the suppression arises from a combination of factors, among which is cancellation between neutron and proton matrix elements because of the isoscalar nature of both the 10.6 MeV GQR and the $2.6 \mathrm{MeV}$ state. A significant isovector admixture in the GQR would lead to a strong enhancement of this transition ${ }^{3}$. Our data rule out such an admixture. It should be noted that our results on the ground state decay show that compound decay is important for the ISGQR (resulting primarily from unusually small compound neutron widths in $20 \mathrm{sp}$ ). It would be reasonable to assume that fully damped states are also important in decays to excited states. The suppression of E1 transitions to the $3^{-}, 2.6 \mathrm{MeV}$ state must therefore survive the damping process, indicating that the compound states into which the GQR mixes retain the isoscalar character of the GQR doorway. The calculations 2,3 illustrating the sensitivity of the gama branch from GQR states in $208 \mathrm{~Pb}$ to the $2.6 \mathrm{MeV} \mathrm{3-}$ state to the isospin character of the GQR state was a major motivation for the 84 MeV/nucieon 170 scattering experiments discussed earlier. These calculations suggested that the strong branch 
to the $3^{-}$state might be an effective way to isolate the IVGQR in 20 ab. Our initial GANIL experiments were a preliminary test. A longer run to study the IVGQR in this way will be carried out this fall. The comparative shurtage of information concerning the isovector GR is largely because of the weakness of isovector excitation in hadron inelastic scattering. The results of the preliminary $84 \mathrm{MeV} / \mathrm{nucleon}$ run clearly show how the increasing importance of coulomb excitation (which is indifferent to isospin) for higher energy heavyion scattering changes this situation. inis is further illustrated by figure 12 which shows the evolution in cross sections in $200 \mathrm{~Pb}$ for the ISGQR, IVGQR, and IVGDR as a function of ${ }^{17} 0$ bombarding energy. There is at least some hope for sensitivity to the IVGQR at GANIL energies, though higher energies would ie preferable.

Figure 13 shows the strength distribution for the IVGQR in $208 \mathrm{~Pb}$ calculated by Bortignon et a.$^{20}$. The dashed 1 ine in the same figure shows ine expected distribution of IVGQR cross section for the $208 \mathrm{~Pb}\left({ }^{17} \mathrm{O},{ }^{17} \mathrm{O}^{1}\right)$ reaction at 84 $\mathrm{MeV} / \mathrm{nucleon}$, resulting from the strong energy dependence of the coulomb excitation process. The yield of $\gamma-\gamma$ roincidences involving transitions through the $2.6 \mathrm{MeV}, 3^{-}$stite should be roughly proportional to the product of these two curves. Shown in figure 14 is the corresponding $\gamma-\gamma$ coincidence yield as a function of excitation energy $\left(E^{\star}\right)$ in ${ }^{208} \mathrm{~Pb}$, where $E_{\gamma_{1}}+E_{\gamma_{2}} \approx E^{\star}$ and $E_{\gamma_{1}}=2.6 \mathrm{MeV}$. In spite of the very poor statistics this result is at least tantalizing.

In conclusion we believe that gamma decay studies can make a significant contribution to our knowledge of the structure of giant resonances. Heavy ion inelastic scatiering followed by gamma decay will become an increasingly important tool at higher bombarding energies. Even for the IVGDR the very large cross sections which can be obtained, and the well understood properties of the dominant coulomb excitation process should soon supplement or perhaps even improve upon results obtained from photon scattering experiments.

It should be noted that people other than the three authors contributed significantly to the various experiments described here. They include D. J. Horen, J. Gomez del Campo, R. L. Auble, B. L. Burks, M. L. Halbert, D. C. Hensley, and R. L. Robinson from Oak Ridge; W. Mittig and $Y$. Schutz from GANIL; B. Haas and J. P. Vivien of Strasbourg, and J. Barrette, N. Alamanos, F. Auger, B. Fernandez, and A. Gillibert from Saclay. 

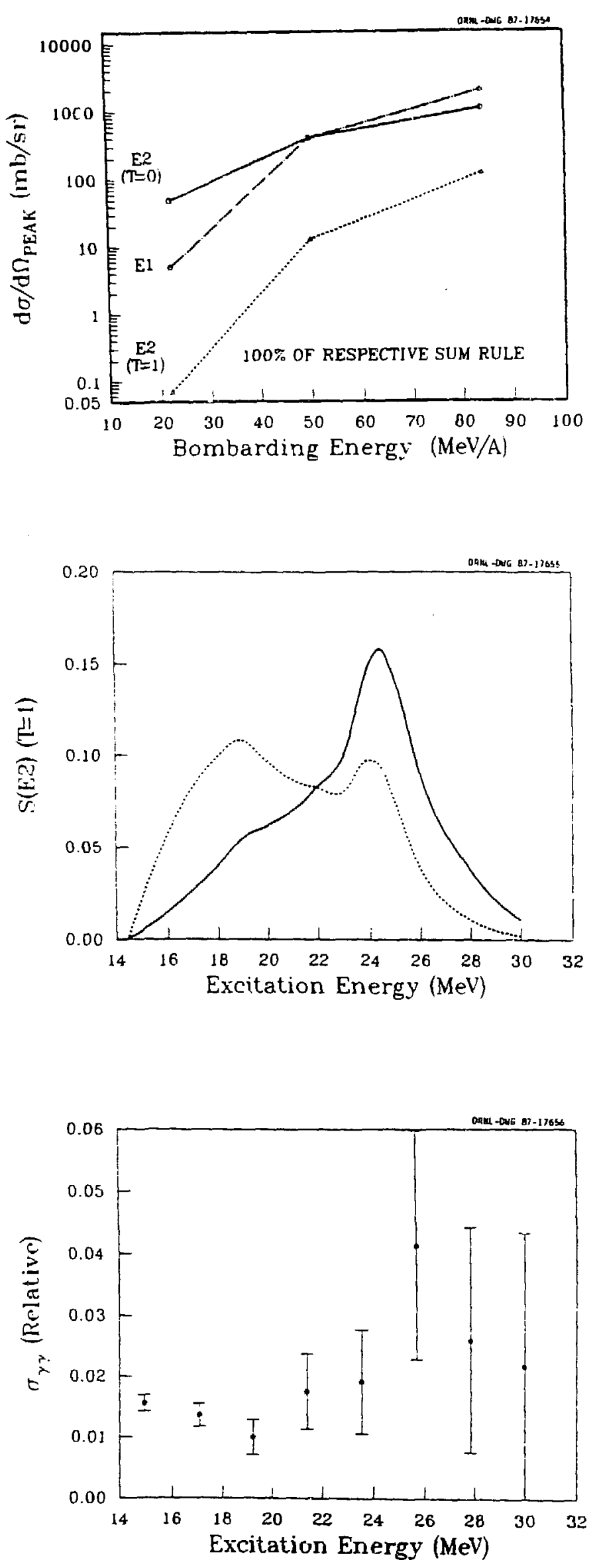

FIGURE 12

The peak differential cross section for inelastic scattering of ${ }^{17} \mathrm{O}$ from ${ }^{208 \mathrm{~Pb}}$ to the resonance states indicated as a function of beam energy.
FIGURE 13

The calculated isovector quadrupole strength function in $20 \mathrm{~Pb}$ (Ref. 20). The dashed curve is the corresponding distribution of inelastic cross section for 84 $\mathrm{MeV} /$ nucleon ${ }^{17} \mathrm{O}$ scattering on $208 \mathrm{~Pb}$.

\section{FIGURE 14}

The relative distribution of $\gamma \gamma$ coincidence yieid as a function of excitation energy, subject to the conditions discussed in the text. 


\section{REFERENCES}

1) F. E. Bertrand, J. R. Beene, and D. J. Horen, "Heavy Ion Excitation of Gtant Resonances - The Next Generation," these proceedings.

2) P. F. Bortignon, R. A. Broglia, and G. F. Bertsch, Phys. Lett. 148B (1984) 20.

3) J. Speth et al., Phys. Rev. C 31 (1985) 2310.

4) J. Barrette et al., submitted for publication.

5) J. Raynal, Phys. Rev. C 23 (1981) 2571.

6) G. R. Satchler, Direct Nuclear Reactions (Oxford University Press, Oxford, 1983), and Phys. Rev. C (in press).

7) H. Feshbach, A. Kerman, and S. Koonin, Annals of Fhysics 125 (1980) 429.

8) M. Hussein and K. McVay, Phys. Rev. Lett. 43 (1979) 1645 and H. Dias, M. S. Hussein, and S. K. Adhikari, Phys. Rev. Lett. 57 (1986) 1998.

9) B. L. Berman and 5. C. Fultz, Rev. Mod. Phys. 47 (1975) 713.

10) J. R. Beene et al., Phys. Lett. 164B (1985) 19.

11) H. Dias et al., Phys. Lett. 173B (1986) 335.

12) P. A. Moldauer, Phys. Rev. C 11 (1974) 426, P. Axel et al., Phys. Rev. C 2 (1970) 689, and 3. E. Lynn, Theory of Neutron Resonance Cross Sections (Oxford University Press, Oxford, 1968).

13) J. R. Beene et al., Nuclear Structure, 1985, edited by R. A. Broglia, G. B. Hagentann, and $\bar{B}$. Herskind, P. 5 .

14) J. R. Beene et al., submitted for publication

15) F. E. Bertrand et al., Phys. Rev. C 34 (1986) 45.

16) H. P. Morsch et a1., Phys. Rev. C 28 (1983) 1947.

17) G Kilgus et al., Z. Phys. A326 (1987) 41.

18) S. J. Seestrom-Morris et al., Phys. Rev. C 33 (1986) 1847.

19) P. R. Bortignon, private communication and contribution to this conference. 